\title{
Different Effects of Phase Advance and Delay in Rotating Light-Dark Regimens on Clock and Natriuretic Peptide Gene Expression in the Rat Heart
}

\author{
I. HERICHOVÁ ${ }^{1}$, J. AMBRUŠOVÁ ${ }^{1}$, L. MOLČAN ${ }^{1}$, A. VESELÁ ${ }^{1}$, P. SVITOK ${ }^{1}$, \\ M. ZEMAN ${ }^{1}$ \\ ${ }^{1}$ Department of Animal Physiology and Ethology, Faculty of Natural Sciences, Comenius \\ University in Bratislava, Bratislava, Slovak Republic
}

Received March 14, 2014

Accepted August 10, 2014

\begin{abstract}
Summary
Under physiological conditions the mammalian circadian system is synchronized to a cyclic environment. The central oscillator in the suprachiasmatic nuclei ( $\mathrm{SCN}$ ) responds predominantly to an external light (L) dark (D) cycle. Peripheral oscillators are more efficiently synchronized by metabolic cues. When the circadian system is exposed to opposing synchronizing cues, peripheral oscillators uncouple from the SCN. To consider influence of phase advances and delays in light regimens mimicking shift work, we analyzed the expression of clock genes (per2, bmal1) and natriuretic peptides (anp, bnp) in the heart of male rats. Experimental groups were exposed to a rotating LD regimen with either $8 \mathrm{~h}$ phase advance or delay for 11 weeks. Samples were taken for a $24 \mathrm{~h}$ cycle in $4 \mathrm{~h}$ intervals. Peripheral oscillators responded to rotating phase advance by decreasing rhythm robustness, while phase delay mostly influenced the phase angle between the acrophase of rhythmic gene expression and the external LD cycle. The expression of anp was arrhythmic in the heart of control rats and was not influenced by rotating LD regimens. The expression of bnp showed a daily rhythm with a nadir during the active phase. The daily rhythm in bnp expression diminished under rotating LD regimen conditions.
\end{abstract}

\section{Key words}

per2 $\bullet b m a l 1 \bullet r e v-e r b a \bullet b n p \bullet a n p \bullet$ Shift work

\section{Corresponding author}

I. Herichova, Department of Animal Physiology and Ethology, Faculty of Natural Sciences, Comenius University Bratislava, Mlynska dolina B-2, 84215 Bratislava, Slovak Republic.

Fax: 00421265429 064. E-mail: herichova@fns.uniba.sk

\section{Introduction}

The circadian system developed during evolution as a genetically conserved adaptation to the regular cycle of the Earth's rotation around its axis (Aschoff et al. 1981). It generates and synchronizes endogenous circadian rhythms to external cycles to predict and better accommodate to expected changes. The circadian system in mammals is hierarchically organized. The central oscillator is located in the suprachiasmatic nuclei of the hypothalamus (SCN) and is synchronized predominantly by external L (light) D (dark) cycles via the retinohypothalamic tract from the retina (Rusak and Zucker 1979). Peripheral oscillators are localized in all other tissues, including other brain tissues, and can be synchronized by neural and humoral inputs from the SCN (Guo et al. 2005).

Both central and peripheral components of the circadian system are composed from autonomous unicellular circadian oscillators. The molecular basis of the generation of mammalian circadian rhythms is a feedback loop created by positive and negative components that influence the transcription of clock genes. Clock genes per and cry possess the capacity to inhibit their own transcription and therefore represent a negative component of the molecular loop. There are three homologous per genes (1,2 and 3) and two homologous cry genes ( 1 and $2)$. The deletion of these genes from the genome causes arrhythmicity under constant conditions. Transcriptional factors BMAL1, CLOCK and NPAS2 represent positive regulators and induce the expression of per and cry genes 
via the E-box regulatory region (Lowrey and Takahashi 2011). In addition to this set of genes, several others were proposed to play an important role in adjusting the basic molecular feedback loop. The nuclear receptor reverse erythroblastosis virus (REV-ERB) is activated by the heterodimer BMAL1:CLOCK via the E-box and feeds back via the REV-ERB/ROR response element (RORE). REV-ERB competes with the stimulatory factor ROR for binding on the RORE element in the promoter region of BMAL1 and NPAS2, and inhibits their expression (Ripperger et al. 2011). Details regarding the function of the core feedback loop, additional modulatory loops and many newly revealed post-transcriptional and posttranslational modifications have been described elsewhere (Albrecht 2012).

Circadian regulation is mediated downstream from the basic molecular feedback loop via clock controlled genes. There are hundreds of rhythmically expressed genes in peripheral tissues (Panda et al. 2002, Bray et al. 2008). In many cases, this rhythmic transcription is mediated via E-box, as it is for per and cry genes. In some cases, the regulation of rhythmic compounds is controlled by other or unknown way.

Atrial natriuretic peptide (ANP) and brain natriuretic peptide (BNP) are produced by the heart and their production increases under conditions of heart failure, myocardial infarction, hypertension, left ventricular hypertrophy and pulmonary hypertension (Nishikimi et al. 2011). Their function is to increase electrolyte and water excretion in the kidney, regulate permeability of the systemic vasculature, and influence proliferation and cardiac hypertrophy. ANP shows a distinct daily rhythm in the plasma of humans (Portaluppi et al. 1990, Goetze et al. 2012), while BNP was not proved to exhibit a daily rhythm in healthy human volunteers (Goetze et al. 2012). On the other hand, expression of bnp mRNA shows a daily rhythm under synchronized (Herichova et al. 2013) and constant darkness conditions (Goetze et al. 2010) in the left ventricle of normotensive rats. Rhythmicity in expression of anp in the rat atria and ventricles showed a borderline significance or arrhythmicity (Goetze et al. 2010, Young et al. 2001). Upon the appropriate signal, ANF and BNP are rapidly released from pre-stored granules. Release of ANP and BNP in response to heart pathology occurs in parallel to a large extent (Sergeeva and Christoffels 2013), and is regulated predominantly via mechanical stress, growth factors and G-protein-coupled receptors (e.g. catecholamines and angiotensin II) (Ma et al. 2005,
Sergeeva and Christoffels 2013). The circadian regulation of anp and bnp expression has not been studied extensively. To our knowledge, functional E-box with a palindromic canonical sequence of CACGTG has not yet been described in the sequences of anp and bnp, although information regarding the presence of CANNTG E-boxes does exist (Thattaliyath et al. 2002, Luo et al. 2006, Wilhide and Jones 2006).

Recent industrial lifestyle changes together with significant light contamination have brought about substantial changes in the organization of a 24-h day. The capacity of peripheral and central oscillators for entrainment by synchronizing cues differs. Light synchronizes the SCN effectively within a day but its influence on clock gene expression in the periphery is rather small (Dibner et al. 2010). Peripheral oscillators can disconnect from central oscillators under conditions of food restriction cycle, which is a weak Zeitgeber for the SCN under synchronized conditions (Damiola et al. 2000). Cycles of food restriction can influence central oscillators under very specific conditions (Nováková et al. 2011, Challet and Mendoza 2010). Food reward is also able to influence the functioning of the circadian system, but this influence is less pronounced compared with food restriction (Challet and Mendoza 2010). All these factors (and many others) influence humans under conditions of shift work and signals to the circadian system become confusing. Deregulation of the circadian system after longterm exposure to irregular LD cycles ( $\geq 10$ years) can promote the progression of some diseases. Epidemiological studies implicate an increased incidence of metabolic disturbances, cardiovascular pathologies and facilitation of carcinogenesis (Haus and Smolensky 2006).

Industrialization has become an indispensable part of life and its risks are understood. Optimization of signals to the circadian system is vital to ensure this evolutionary adaption remains beneficial, or at the very least, to ensure consequences of internal desynchronization are prevented (Saksvik et al. 2011, Herichova 2013). Understanding of entrainment by light regimen and food intake provides a good experimental basis for studying which schedules should be used in shift work and identifying schedules less deleterious for the functioning of the circadian system.

Therefore, the aim of this study was to analyze the effects of LD schedules used during rotating shift work with phase advances or phase delays on clock genes and ANP and BNP mRNA in the rat heart, and compare the effects of these schedules on peripheral oscillators in the heart. 


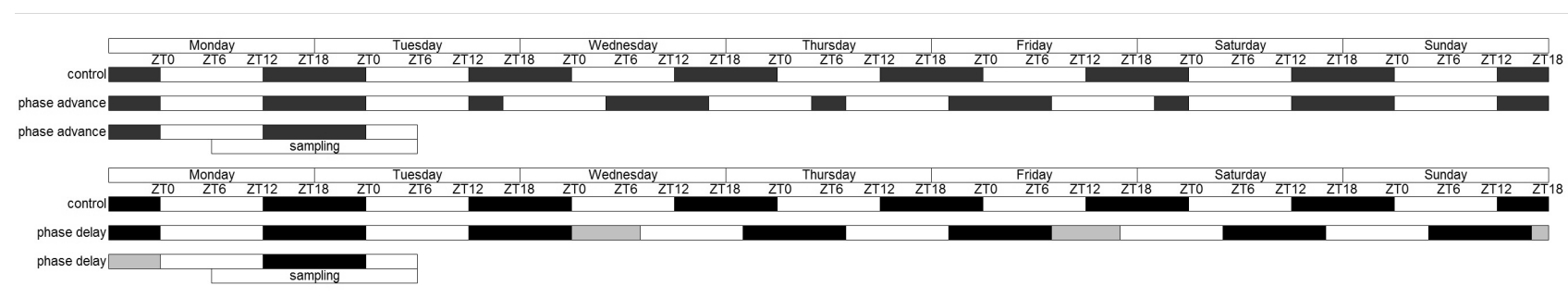

Fig. 1. Scheme of rotating light:dark regimen with phase advances (upper panel) or phase delays (lower panel). ZT - Zeitgeber time. Black bars indicate the dark part of the $24 \mathrm{~h}$ cycle. Gray bars indicate delay in rotating regimen.

\section{Methods}

Male Wistar rats $(n=145)$ were obtained from Anlab Praha (Czech Republic) at 5 weeks of age with an initial weight of $139.4 \pm 2.1 \mathrm{~g}$. Animals were housed in temperature-controlled rooms $\left(21 \pm 2{ }^{\circ} \mathrm{C}\right)$ under a $12: 12$ LD cycle. Food and water were available ad libitum. The study was performed in two consecutive phases with two independent control groups. The effects of phase advance were investigated in 'Experiment 1', and the influence of phase delay was analyzed in 'Experiment 2'. After an acclimatization period, experimental groups were exposed to a LD regimen mimicking shift work with 8-h phase advances (the dark phase of the $24 \mathrm{~h}$ cycle was shortened by $8 \mathrm{~h}$ three times per week over a period of 12 weeks) or delays (the dark phase of the $24 \mathrm{~h}$ cycle was lengthened by $8 \mathrm{~h}$ every second day over a period of 11 weeks) (Fig. 1). Blood pressure was measured during the whole experiment using the tail-cuff plethysmography method (AD Instruments, Germany).

Locomotor activity was measured in seven rats from Experiment 1 and in four rats from Experiment 2 by radiotelemetry as described previously (Molcan et al. 2013). From 15-min averaged segments of acquired data, double-plot actograms were created using Chronos-Fit (Zuther et al. 2009).

At the end of the experiment sampling was performed in $4 \mathrm{~h}$ intervals during a $24 \mathrm{~h}$ cycle when the LD cycles of the control and experimental groups were the same for 1 day (Fig. 1). Prior to sampling rats were anaesthetized by carbon dioxide and subsequently decapitated $(n=4-8)$. The heart was weighed and samples were taken within a few minutes, snap frozen in liquid nitrogen and stored under $-80{ }^{\circ} \mathrm{C}$ until RNA isolation. The experimental protocol was approved by the Ethical Committee for the Care and Use of Laboratory Animals at Comenius University Bratislava (Slovakia) and the State Veterinary Authority.

Total RNA from the heart was isolated with the use of TRI Reagent ${ }^{\circledR}$ (Total RNA Isolation reagent, MRC, USA). RNA integrity and contamination with DNA were examined using $1.5 \%$ agarose $/ 10.7 \mathrm{M}$ formaldehyde gel (Herichova et al. 2001). First-strand cDNA synthesis was carried out with the use of the ImProm-II ${ }^{\mathrm{TM}}$ Reverse Transcription System (Promega, USA) according to the manufacturer's instruction. Quantification of cDNA was performed by real-time PCR using the QuantiTect SYBR $^{\circledR}$ Green PCR kit (Qiagen, Germany) and StepOne ${ }^{\mathrm{TM}}$ System (Applied Biosystems, USA). The primer pairs used for the amplification of per2, bmall, rev-erba, bnp, gapdh and rplp1 and realtime PCR conditions were as described in previous reports (Szántóová et al. 2011, Herichova et al. 2013). The other primer pairs used for the amplification were: anp (NM_012612) sense 5'-TCA AGA ACC TGC TAG ACC A-3', antisense 5'-TCT GAG ACG GGT TGA CTT CC-3' (annelation $49^{\circ} \mathrm{C}$ ). Gene expression was normalized to rplpl or gadph. The specificity of the PCR reaction was validated by melting curve analysis. The fluorescence dye ROX served as an internal reference for normalization of the SYBR Green I fluorescent signal.

\section{Statistical evaluation}

To compare gene expression between two groups an unpaired t-test was used, and ANOVA followed by Tukey's post-hoc test was used to compare gene expression between more than two groups. To analyze the daily profiles of clock genes, expression data were fitted into a cosinor curve $24 \mathrm{~h}$ period (Nelson et al. 1979, Klemfus and Clopton 1993). When experimental data significantly matched the cosinor curve, the following parameters were calculated with a confidence limit of $95 \%$ : mesor (the time series mean), amplitude (one half of the peak-trough difference expressed herein relative to the mesor) and acrophase (peak time referenced to the time of lights on in the animal facility). Goodness of fit ( $\mathrm{R}$ value - correlation coefficient) of the approximated curve was estimated by ANOVA. 
Confidence limits of rhythm parameters were calculated by SigmaPlot (USA). Time is expressed in Zeitgeber time (ZT), when ZT0 is defined as the beginning of the light part of the $24 \mathrm{~h}$ cycle. Circadian and ultradian rhythm analysis of the individual locomotor activity was performed with a Lomb-Scargle periodogram using Chronos-Fit software (Zuther et al. 2009, Molcan et al. 2013). In the actograms, the black bars indicate animal activity, each line represents 1 day of experiment and recordings are double plotted. Data in graphs displaying gene expression are presented as the arithmetic mean and standard error of the mean.

\section{Results}

Exposure of rats to rotating $\mathrm{LD}$ regimens for 11 weeks did not cause an increase in blood pressure or heart hypertrophy in the experimental groups compared with the corresponding control group. Furthermore, we did not observe a significant change in body weight compared with the control (data not shown).
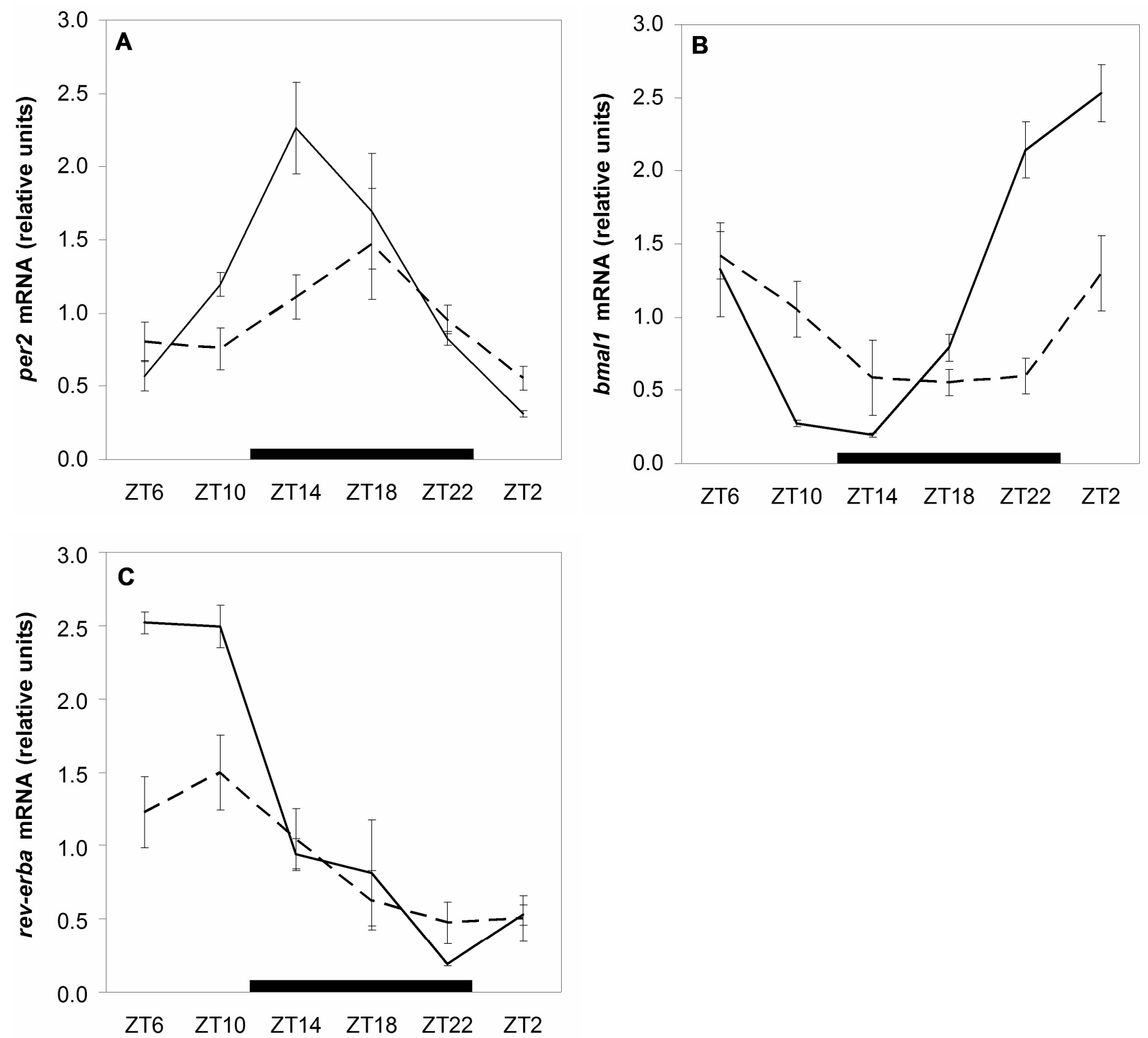

Fig. 2. Effect of rotating light:dark regimen with phase advances on per2 (A), bmal1 (B) and rev-erba (C) mRNA expression in rat hearts. The solid line represents the control group and the broken line shows data from the experimental group $(n=4-8)$. The black bar on the bottom of the graph represents the dark part of the $24 \mathrm{~h}$ cycle. Time scale is given in relative units: Zeitgeber time (ZTO = beginning of the light part of the light:dark cycle). 

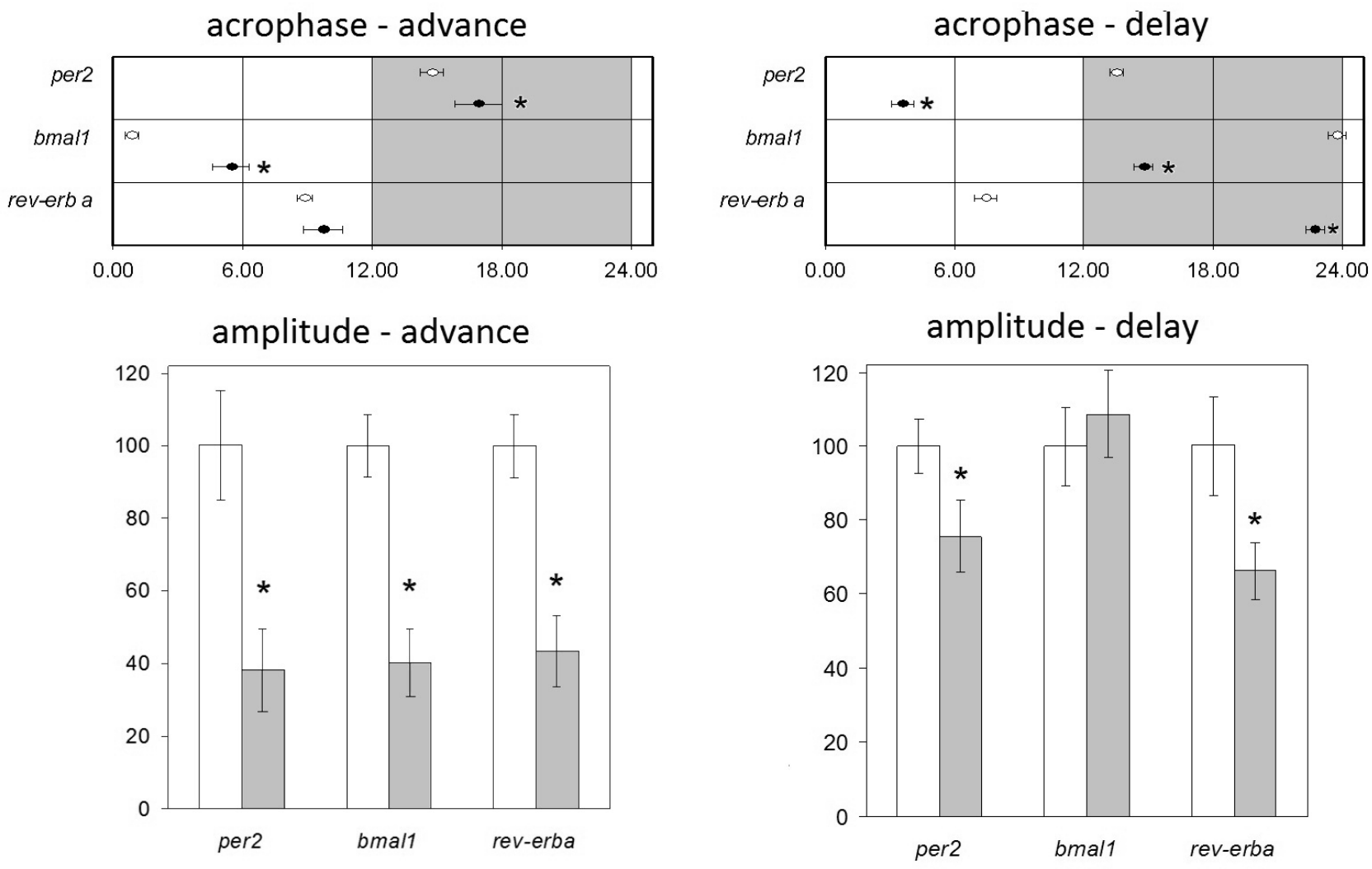

Fig. 3. Effect of direction of light:dark regimen rotation on the amplitude and acrophase of rhythmic profiles of per2, bmal1 and reverba mRNA expression in the heart. The white circles and columns represent the control groups. The black circles and gray columns correspond to data measured in rats exposed to rotating light:dark regimens. Amplitude is expressed as a relative value assuming calculated amplitude of the control group to be $100 \%$ (control of Experiment 1 or 2 depending on regimen). Differences in acrophase and amplitude induced by rotating phase delay were calculated from a previous study (Szántóová et al. 2011). * P<0.05 cosinor (Table 1).

Rotating regimen with phase advances caused a significant decrease in mesor and amplitude of per 2 expression and the per 2 acrophase was shifted by $2 \mathrm{~h}$ compared with the control (Figs 2A, 3; Table 1).

The rotating phase advance LD regimen strongly influenced the daily profile of bmall expression. The expression of bmall showed a significant decrease in mesor and amplitude, and the acrophase shifted by $4 \mathrm{~h}$ compared with the control (Figs 2B, 3; Table 1).

Expression of rev-erba was significantly suppressed in the group exposed to the LD regimen with rotating advances compared with the control. The daily profile of rev-erba showed a decreased mesor and amplitude with no significant influence on the acrophase (Figs 2C, 3; Table 1) compared with the control group.

The effects of phase delays and advances on rhythmic clock genes in the heart were compared, and the effect of rotating LD regimen with phase delays on clock gene expression was calculated from our previous work (Szántóová et al. 2011). The effects of phase advance and phase delay on per2, bmall and rev-erba expression were very different. The LD regimen with rotating phase advance had a much stronger effect on amplitude and less pronounced effect on acrophase compared with the LD regimen with rotating phase delays (Fig. 3).

The locomotor activity of animals was monitored by individual actograms (Fig. 4). During the first week of monitoring under a standard 12:12 LD regimen, all animals displayed a typical circadian pattern (period $\sim 24 \mathrm{~h}, \mathrm{P}<0.0001$ ). The effect of a rotating LD regimen with phase advance was analyzed in seven rats (Experiment 1). At the end of the experiment four animals still maintained a periodicity of $\sim 24 \mathrm{~h}$ period $(\mathrm{P}<0.001)$, although with decreased significance in three of them. Two animals displayed only periodicities shorter than $24 \mathrm{~h}$, and one animal acquired several shorter and one longer period compared with the control profile. There was an increase in ultradian components occurrence and activity during the light part of LD regimen and in six animals. Under conditions of a rotating $\mathrm{LD}$ regimen with phase delays we observed an increase in periodicity $(27-29 \mathrm{~h}, \mathrm{P}<0.001)$ in all four 


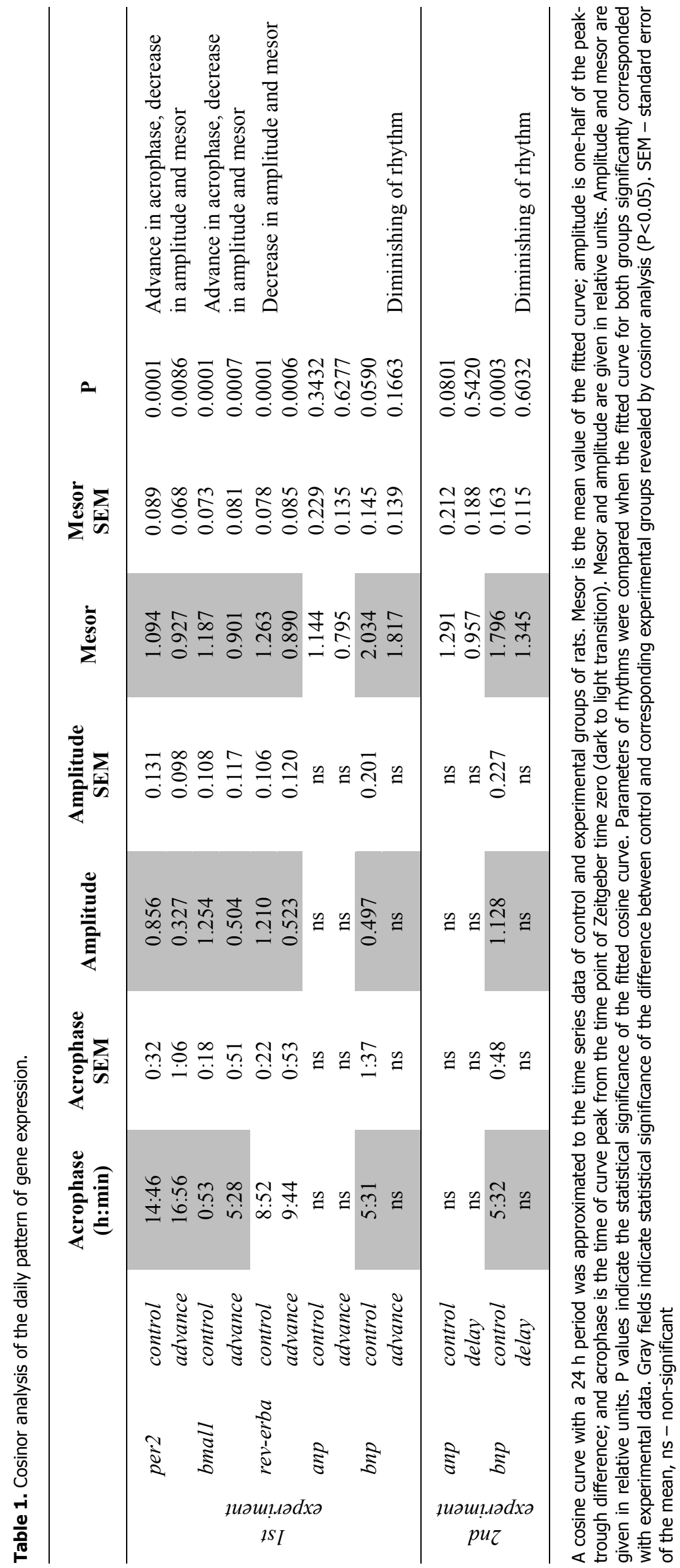


animals and the appearance of several periodicities with a shorter duration (Experiment 2). According to our results the response of animals was more homogenous in group exposed to rotating delays compared with backward shifts.

\section{Advance}

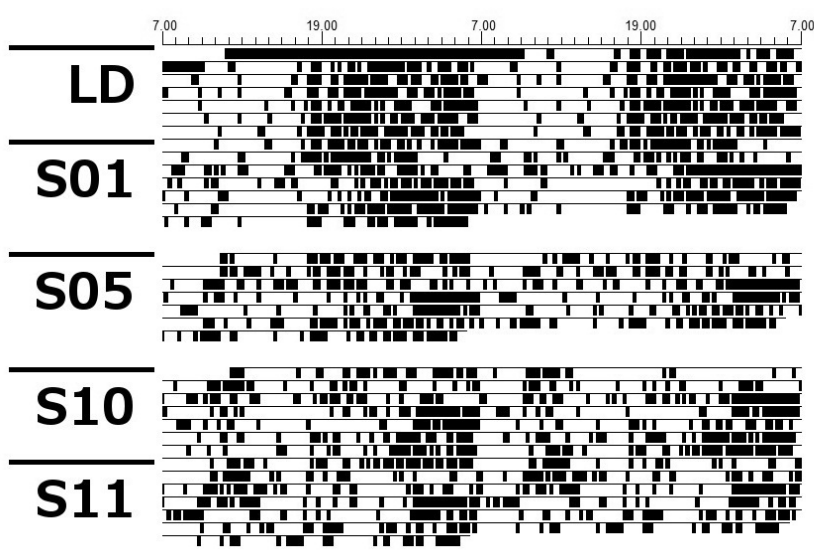

Delay

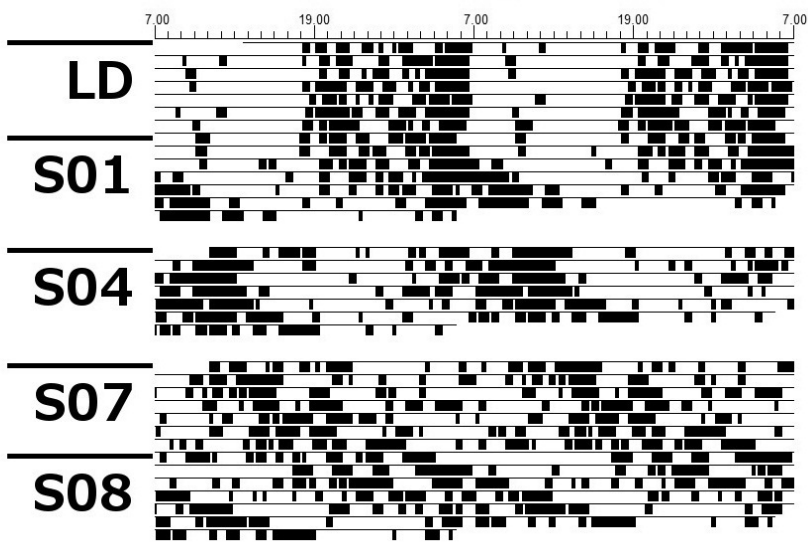

Fig. 4. Representative double-plotted actograms showing rat locomotor activity rhythm during 1 week of light:dark conditions followed by 11 weeks of phase advance shifts of light or 8 weeks of phase delay shifts of light. LD - regular cycle 12 light:12 dark; S - regimen with rotating light:dark cycle. Number indicates the duration of experiment at the time of recording.

To evaluate the effect of rotating LD regimens on the heart, we measured the expression of natriuretic peptides anp and bnp that closely parallel many pathological states of the cardiovascular system. A significant rhythm in anp expression was not observed in the left ventricle of the control and experimental groups (Fig. 5A,C; Table 1). A decreasing trend in anp expression between control and experimental groups was observed in both experiments but did not reach a level of significance (t-test: $\mathrm{P}=0.136$ Experiment $1 ; \mathrm{P}=0.282$ Experiment 2). ANOVA did not show significant differences between anp mRNA expression at six time points of a $24 \mathrm{~h}$ cycle in control groups (Experiment 1 anp control $\mathrm{F}(5,28)=0.468, \mathrm{P}=0.797$; Experiment 2 anp control $\mathrm{F}(5,18)=1.446, \mathrm{P}=0.256$ ). Differences in the expression of anp in the heart of rats exposed to phase shifts at six time points of Experiment 1 (anp advance $\mathrm{F}(5,29)=0.218, \mathrm{P}=0.952)$ and Experiment 2 (anp delay $\mathrm{F}(5,17)=0.279, \quad \mathrm{P}=0.918)$ were not detected. Taken together, no significant relationship between the LD regimens and anp expression was observed under these experimental conditions.

The significance of rhythmicity in bnp expression was borderline in the control group of Experiment 1 (Fig. 5D; Table 1). A very distinct daily pattern was observed in the control group of
Experiment 2 (Fig. 5B; Table 1). In both cases a daily rhythm peaked at ZT5 during the passive phase of the $24 \mathrm{~h}$ rhythm. ANOVA confirmed the differences between time points in the control group of Experiment 2 (bnp control $\mathrm{F}(5,17)=5.231, \mathrm{P}=0.004)$ and the trend in Experiment 1 (bnp control $\mathrm{F}(5,28)=2.063, \mathrm{P}=0.100$ ). A rhythmic pattern in bnp expression was not observed in experimental animals exposed to either rotating advances or delays (Table 1). ANOVA did not indicate any differences among time points in the experimental groups (Experiment 1 bnp advance $\mathrm{F}(5,29)=0.787, \mathrm{P}=0.567$; Experiment 2 bnp delay $\mathrm{F}(5,17)=1.711, \mathrm{P}=0.186)$.

The mRNA expression of natriuretic peptides did not correlate with blood pressure under normotensive conditions in either the control or experimental groups. Furthermore, no significant correlation between natriuretic peptide mRNA expression and heart/body weight index was observed in the control or experimental groups (data not shown).

In both control groups, the expression of anp and bnp correlated together during the light part of the $24 \mathrm{~h}$ cycle (control Experiment 1: $y=1.7083 x-2.3936$, $\mathrm{R}=0.852, \mathrm{n}=12$; control Experiment 2: $\mathrm{y}=1.3897 \mathrm{x}-$ 344.48, $\mathrm{R}=0.607, \mathrm{n}=16$ ). anp and bnp expression did not correlate during the light part of the $24 \mathrm{~h}$ cycle in animals exposed to rotating $\mathrm{LD}$ regimens. 

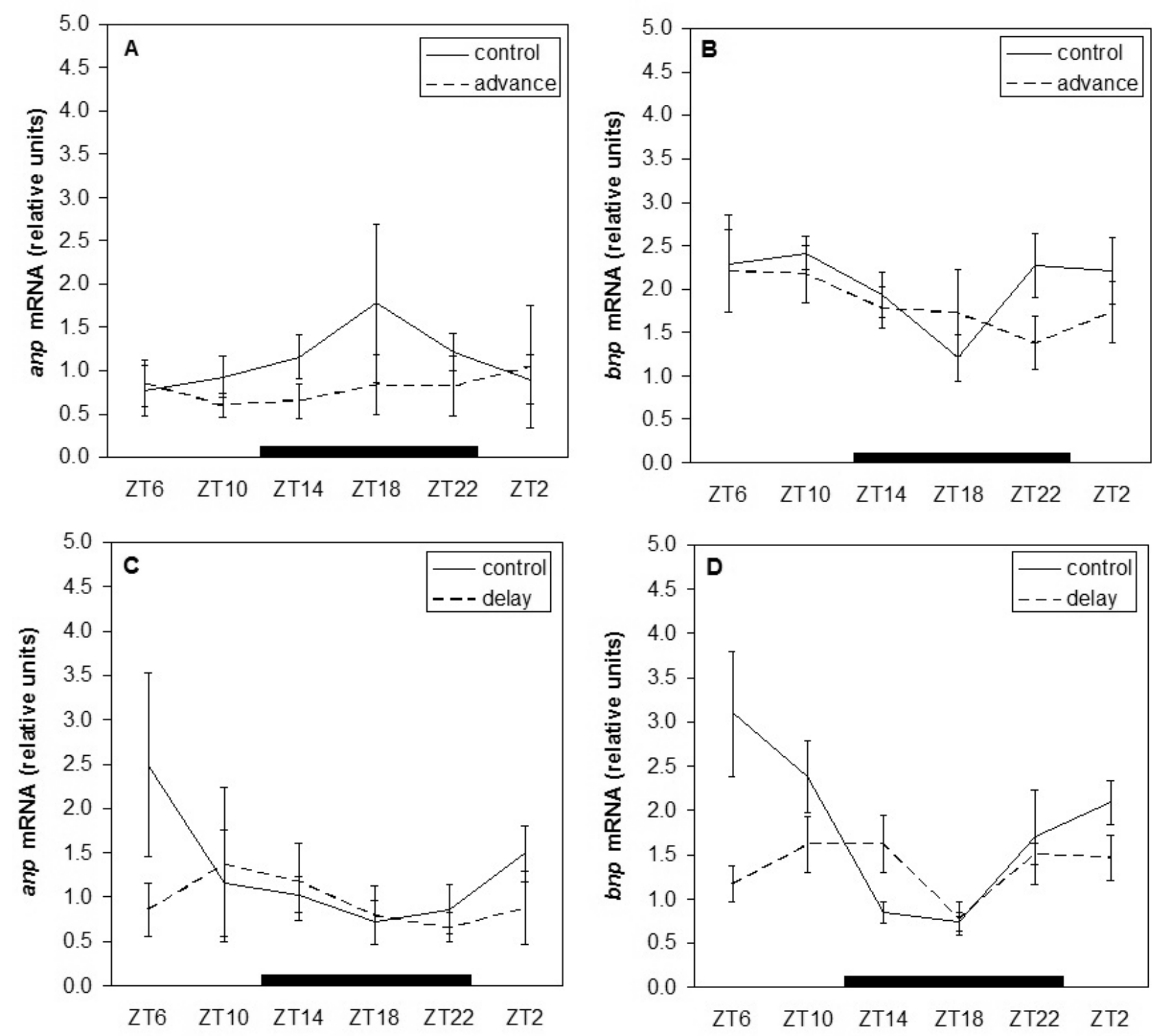

Fig. 5. Effect of rotating light:dark regimen with phase advances or phase delays on $\operatorname{anp}(\mathbf{A}, \mathbf{C})$ and $b n p(\mathbf{B}, \mathbf{D})$ mRNA expression in the hearts of rats. The solid line represents the control group and the broken line shows data from the experimental group ( $n=3-8)$. The black bar on the bottom of the graph represents the dark part of the $24 \mathrm{~h}$ cycle. Time scale is given in relative units Zeitgeber time (ZTO = beginning of the light part of the light:dark cycle).

\section{Discussion}

The aim of this study was to elucidate the physiological principles of uncoupling of peripheral oscillators from the central oscillator under conditions of rotating LD regimens. According to the classical rules of entrainment, the strength of Zeitgeber depends upon its robustness and angle difference (Aschoff et al. 1981).

This study indicates that under conditions of rotating $\mathrm{LD}$ regimen with phase delays robustness of the molecular oscillator is preserved, but the oscillator is out of phase with the ambient LD cycle. Under conditions of rotating $\mathrm{LD}$ regimen with phase advances oscillations of rhythmic clock gene expression are maintained and acrophase is similar to the control group but with less robustness. Both phase advances and phase delays resulted in weakened regulation of clock controlled genes by the central loop, but via different way.

The daily profiles of per2, bmall and rev-erba mRNA observed in the control groups showed the same pattern as it was published previously (Young et al. 2001, Szántóová et al. 2011, Herichova et al. 2013). The application of phase-advanced rotating LD cycles resulted in a pronounced decrease in the amplitude of per 2 and bmall rhythmic expression (down to $40 \%$ ), while phase-delayed rotating LD cycles caused a phase 
delay in the acrophase (by 8-9 h) but the change in amplitude was much smaller.

Rev-erba (NR1D1) encodes an orphan member of the nuclear receptor superfamily and is a clock controlled gene. The basic molecular loop influences reverba via E-boxes in its promoter region and the rhythmic pattern of rev-erba expression is impaired in clock mutant mice (Triqueneaux et al. 2004). Rhythmic expression of rev-erba mRNA showed a significant decrease in amplitude under both LD regimens, but the difference was more pronounced in animals exposed to the LD regimen with rotating advances. The acrophase of the rhythmic expression of rev-erba was significantly influenced by rotating phase delays. Rotating phase advances did not cause a significant change in rev-erba acrophase. This suggests that the rhythmic expression of rev-erba is less disturbed in animals exposed to phase advance rotating $\mathrm{LD}$ regimen since under these conditions it corresponds better to actual LD regimen. It is possible that lower robustness of per 2 and bmall expression results in weakened circadian regulation of rev-erba and that other regulatory regions of the rev-erba gene or protein contribute to its regulation to a greater extent (Duez and Staels 2009).

The activity records of three of the four animals exposed to rotating phase delays showed a substantial and significant increase in period length. The fourth rat also displayed a periodicity of $\sim 29 \mathrm{~h}$, but component was the second most powerful. The response of animals exposed to rotating $\mathrm{LD}$ regimen with phase delays was more homogenous compared with those exposed to phase advances. In the animals exposed to backward shifts, four animals maintained a significant periodicity of $\sim 24 \mathrm{~h}$. All animals in Experiment 1 displayed an increase in the presence of ultradian, and in two cases prolonged periodicities with high individual variability.

Actograms and data obtained at the level of gene expression cannot be linked directly. Some parallel can be seen in Experiment 1 where the amplitude of rhythm in locomotor activity as well as gene expression is lowered. The circadian regulation of locomotor activity and gene expression seem to more persistently take after rotating LD regimen with phase delays compared with phase advances.

During shift work many environmental and individual factors play a role in the final response of the organism (Saksvik et al. 2011). Together with changes in LD regimen also a food intake is changed and this factor is particularly important in the synchronization of peripheral oscillators (Damiola et al. 2000). Peripheral oscillators in the heart can be synchronized to food regimen when the $\mathrm{LD}$ cycle is reversed, although it is the influence of both of these factors (LD cycle and food cycle) that significantly accelerates this process (Wu et al. 2008, 2010). It was showed that reversed LD cycle lasting for 1 week does not significantly influence the acrophase of bmall, per1, cryl and decl in the heart of rats with no access to food during the dark phase (Wu et al. 2008). However, reversed accessibility to food inverted the acrophase of clock genes within 7 days, even when the rat could only access food during the light phase. If the effect of food availability is strengthened by the LD cycle, synchronization occurs within 5 days. Expression of rev-erba, which is proposed to play a role as a metabolic sensor of the circadian system (Duez and Staels 2009), is able to synchronize to reversed food regimen strengthened by the $\mathrm{LD}$ cycle in 5 days (Wu et al. 2010). The role of food availability was implicated in a model of metabolic desynchrony in rats with forced activity during the passive phase and food availability during the active phase of the LD cycle (SalgadoDelgado et al. 2010). The study demonstrated that the time of food consumption was a more crucial factor in the generation of metabolic disturbances than forced activity. In our study, food intake to some extent corresponded with the presented actograms as food was available ad libitum. We suppose that food intake is important factor in the generation of the final pattern of clock gene expression in the heart, and it represents the most appropriate tool for synchronizing peripheral oscillators under conditions of irregular LD cycles.

In the experimental setups in this study, an increase in blood pressure and heart/body weight index was not observed. Under normotensive conditions, anp shows an arrhythmic daily profile and hypertension induces its expression (Young et al. 2001). Our data are in accordance with previous reports. Expression of anp in the heart did not show a daily rhythm and its expression was not increased by rotating LD regimens, presumably because these treatments did not cause hypertension. Expression of bnp shows a daily profile in the heart, as has previously been reported (Goetze et al. 2010, Herichova et al. 2013). In the present study we detected significant daily rhythm in one control group (Experiment 2) and a borderline rhythmic pattern in the control group of Experiment 1, indicating that the daily pattern of bnp expression has a rather low amplitude. Both rotating $L D$ regimens resulted in loss of the 
rhythmic pattern in bnp expression in the heart of experimental rats. This finding suggests that the rhythmic influence of bnp on blood pressure is weakened under conditions of disturbed circadian system. This is also supported by our previous study showing that rats kept under irregular LD conditions have suppressed circadian control of heart rate and blood pressure, and rely more on an acute response to the LD regimen (Molcan et al. 2013).

The question of which direction of rotation is less deleterious for the individual can be elucidated by this study only to a limited extent. Most previous epidemiological and experimental studies indicated that rotating LD regimen with phase advances are more harmful to humans (Saksvik et al. 2011, Deacon and Arendt 1996). In our study, rotating phase delays synchronized peripheral oscillators in the heart to a shifted acrophase and rev-erba expression remained coupled to the basic loop, while bnp expression did not.
Backward rotation, which causes a decrease in the amplitude and rhythmicity of all measured genes, possibly allows the system to be more responsive to acute influences.

To conclude, the observed significant changes demonstrate the two ways by which the circadian system can cope with fast rotating LD regimens. In case of rotating delays, the predictive capacity was preserved but this process was not well timed in respect to the actual LD cycle and in case phase advances we observed a lower robustness, perhaps leading to weaker circadian coordination in the periphery.

\section{Conflict of Interest}

There is no conflict of interest.

\section{Acknowledgements}

Research was supported by grant VEGA 1/1262/12, APVV-0291-12.

\section{References}

ALBRECHT U: Timing to perfection: the biology of central and peripheral circadian clocks. Neuron 74: 246-260, 2012.

ASCHOFF J, KING FA, STRICKER EM: Handbook of Behavioral Neurobiology: Biological Rhythms, Volume 4. Plenum Press, New York, 1981, pp 1-156.

BRAY MS, SHAW CA, MOORE MW, GARCIA RA, ZANQUETTA MM, DURGAN DJ, JEONG WJ, TSAI JY, BUGGER H, ZHANG D, ROHRWASSER A, RENNISON JH, DYCK JR, LITWIN SE, HARDIN PE, CHOW CW, CHANDLER MP, ABEL ED, YOUNG ME: Disruption of the circadian clock within the cardiomyocyte influences myocardial contractile function, metabolism, and gene expression. Am J Physiol 294: H1036-H1047, 2008.

CHALLET E, MENDOZA J: Metabolic and reward feeding synchronises the rhythmic brain. Cell Tissue Res 341: 1-11, 2010.

DAMIOLA F, LE MINH N, PREITNER N, KORNMANN B, FLEURY-OLELA F, SCHIBLER U: Restricted feeding uncouples circadian oscillators in peripheral tissues from the central pacemaker in the suprachiasmatic nucleus. Genes Dev 14: 2950-2961, 2000.

DEACON S, ARENDT J: Adapting to phase shifts, I. An experimental model for jet lag and shift work. Physiol Behav 59: 665-673, 1996.

DIBNER C, SCHIBLER U, ALBRECHT U: The mammalian circadian timing system: organization and coordination of central and peripheral clocks. Annu Rev Physiol 72: 517-549, 2010.

DUEZ H, STAELS B: Rev-erb-alpha: an integrator of circadian rhythms and metabolism. J Appl Physiol 107: 19721980, 2009.

GOETZE JP, GEORG B, JØRGENSEN HL, FAHRENKRUG J: Chamber-dependent circadian expression of cardiac natriuretic peptides. Regul Pept 160: 140-145, 2010.

GOETZE JP, JØRGENSEN HL, SENNELS HP, FAHRENKRUG J: Diurnal plasma concentrations of natriuretic propeptides in healthy young males. Clin Chem 58: 789-792, 2012.

GUO H, BREWER JM, CHAMPHEKAR A, HARRIS RB, BITTMAN EL: Differential control of peripheral circadian rhythms by suprachiasmatic-dependent neural signals. Proc Natl Acad Sci USA 102: 3111-3116, 2005. 
HAUS E, SMOLENSKY M: Biological clocks and shift work: circadian dysregulation and potential long-term effects. Cancer Causes Control 17: 489-500, 2006.

HERICHOVA I: Changes of physiological functions induced by shift work. Endocr Regul 47: 159-170, 2013.

HERICHOVÁ I, ZEMAN M, MACKOVÁ M, GRIAC P: Rhythms of the pineal N-acetyltransferase mRNA and melatonin concentrations during embryonic and post-embryonic development in chicken. Neurosci Lett 298: 123-126, 2001.

HERICHOVA I, ŠOLTÉSOVÁ D, SZÁNTÓOVÁ K, MRAVEC B, NEUPAUEROVÁ D, VESELÁ A, ZEMAN M: Effect of angiotensin II on rhythmic per2 expression in the suprachiasmatic nucleus and heart and daily rhythm of activity in Wistar rats. Regul Pept 186: 49-56, 2013.

KLEMFUSS H, CLOPTON PL: Seeking tau: a comparison of six methods. J Interdisciplinary Cycle Res 24: 1-16, 1993.

LOGAN RW, ZHANG C, MURUGAN S, O'CONNELL S, LEVITT D, ROSENWASSER AM, SARKAR DK: Chronic shift-lag alters the circadian clock of NK cells and promotes lung cancer growth in rats. J Immunol 188: 2583-2591, 2012.

LOWREY PL, TAKAHASHI JS: Genetics of circadian rhythms in Mammalian model organisms. Adv Genet 74: 175$230,2011$.

LUO Y, JIANG C, BELANGER AJ, AKITA GY, WADSWORTH SC, GREGORY RJ, VINCENT KA: A constitutively active hypoxia-inducible factor-1alpha/VP16 hybrid factor activates expression of the human B-type natriuretic peptide gene. Mol Pharmacol 69: 1953-1962, 2006.

MA KK, BANAS K, DE BOLD AJ: Determinants of inducible brain natriuretic peptide promoter activity. Regul Pept 128: 169-176, 2005.

MOLCAN L, TEPLAN M, VESELA A, ZEMAN M: The long-term effects of phase advance shifts of photoperiod on cardiovascular parameters as measured by radiotelemetry in rats. Physiol Meas 34: 1623-1632, 2013.

NELSON W, TONG YL, LEE JK, HALBERG F: Methods for cosinor-rhythmometry. Chronobiologia 6: 305-323, 1979.

NISHIKIMI T, KUWAHARA K, NAKAO K: Current biochemistry, molecular biology, and clinical relevance of natriuretic peptides. J Cardiol 57: 131-140, 2011.

NOVÁKOVÁ M, POLIDAROVÁ L, SLÁDEK M, SUMOVÁ A: Restricted feeding regime affects clock gene expression profiles in the suprachiasmatic nucleus of rats exposed to constant light. Neuroscience 197: 65-71, 2011.

PANDA S, ANTOCH MP, MILLER BH, SU AI, SCHOOK AB, STRAUME M, SCHULTZ PG, KAY SA, TAKAHASHI JS, HOGENESCH JB: Coordinated transcription of key pathways in the mouse by the circadian clock. Cell 109: 307-320, 2002.

PORTALUPPI F, BAGNI B, DEGLI UBERTI E, MONTANARI L, CAVALLINI R, TRASFORINI G, MARGUTTI A, FERLINI M, ZANELLA M, PARTI M: Circadian rhythms of atrial natriuretic peptide, renin, aldosterone, cortisol, blood pressure and heart rate in normal and hypertensive subjects. J Hypertens 8: 85-95, 1990.

RIPPERGER JA, JUD C, ALBRECHT U: The daily rhythm of mice. FEBS Lett 585: 1384-1392, 2011.

RUSAK B, ZUCKER I: Neural regulation of circadian rhythms. Physiol Rev 59: 449-526, 1979.

SAKSVIK IB, BJORVATN B, HETLAND H, SANDAL GM, PALLESEN S: Individual differences in tolerance to shift work - a systematic review. Sleep Med Rev 15: 221-235, 2011.

SALGADO-DELGADO R, ANGELES-CASTELLANOS M, SADERI N, BUIJS RM, ESCOBAR C: Food intake during the normal activity phase prevents obesity and circadian desynchrony in a rat model of night work. Endocrinology 151: 1019-1029, 2010.

SERGEEVA IA, CHRISTOFFELS VM: Regulation of expression of atrial and brain natriuretic peptide, biomarkers for heart development and disease. Biochim Biophys Acta 1832: 2403-2413, 2013.

SZÁNTÓOVÁ K, ZEMAN M, VESELÁ A, HERICHOVA I: Effect of phase delay lighting rotation schedule on daily expression of per2, bmal1, rev-erb $\alpha$, ppara, and pdk4 genes in the heart and liver of Wistar rats. Mol Cell Biochem 348: 53-60, 2011.

THATTALIYATH BD, FIRULLI BA, FIRULLI AB: The basic-helix-loop-helix transcription factor HAND2 directly regulates transcription of the atrial naturetic peptide gene. J Mol Cell Cardiol 34: 1335-1344, 2002. 
TRIQUENEAUX G, THENOT S, KAKIZAWA T, ANTOCH MP, SAFI R, TAKAHASHI JS, DELAUNAY F, LAUDET V: The orphan receptor Rev-erbalpha gene is a target of the circadian clock pacemaker. $J$ Mol Endocrinol 33: 585-608, 2004.

YOUNG ME, RAZEGHI P, TAEGTMEYER H: Clock genes in the heart: characterization and attenuation with hypertrophy. Circ Res 88: 1142-1150, 2001.

WILHIDE ME, JONES WK: Potential therapeutic gene for the treatment of ischemic disease: Ad2/hypoxia-inducible factor-1alpha (HIF-1)/VP16 enhances B-type natriuretic peptide gene expression via a HIF-1-responsive element. Mol Pharmacol 69: 1773-1778, 2006.

WU T, JIN Y, NI Y, ZHANG D, KATO H, FU Z: Effects of light cues on re-entrainment of the food-dominated peripheral clocks in mammals. Gene 419: 27-34, 2008.

WU T, NI Y, ZHUGE F, FU Z: Resetting process of peripheral circadian gene expression after the combined reversal of feeding schedule and light/dark cycle via a 24-h light period transition in rats. Physiol Res 59: 581-590, 2010.

ZUTHER P, GORBEY S, LEMMER B: Chronos-Fit 1.06. 2009. http://www.chronopharmacology.de/software.htm 